\title{
Kératokystes odontogènes multiples non syndromiques : une nouvelle observation
}

\author{
Sofia Haitami* , Hajar Oulammou, Ihsane Ben Yahya \\ Centre de Consultation et de Traitement dentaire, Faculté de Médecine dentaire, Casablanca, Maroc
}

(Reçu le 23 avril 2013, accepté le 2 juillet 2013)

Mots clés : kyste odontogéne / kératokyste / naevomatose basocellulaire / syndrome de Gorlin et Golz

Key words: odontogenic cyst / keratocyst / basal cell nevus syndrome / Gorlin Goltz syndrome

\begin{abstract}
Résumé - Introduction. Le kératokyste odontogénique occupe une partie importante dans le tableau clinique de la naevomatose basocellulaire ou syndrome de Gorlin et Goltz. La mutation du gène PTCH est en partie responsable de ce syndrome, son expression partielle peut être à l'origine de l'apparition de kératokystes multiples récidivants. Observation. On rapporte à travers cet article le cas d'un patient âgé de 54 ans ne présentant aucun symptôme révélateur du syndrome de Gorlin et Goltz mais qui, néanmoins, a développé des kystes multiples intéressant tout le corps de la mandibule. Le traitement a consisté en l'énucléation des cavités kystiques en plusieurs temps, l'examen anatomopathologique des pièces opératoires a confirmé le diagnostic de kératokystes multiples. Le suivi sur 24 mois a montré l'absence de signe de récidive. Discussion. Des kératokytes multiples peuvent quelquefois être observés en dehors de la naevomatose baso-cellulaire.
\end{abstract}

\begin{abstract}
Non-syndromic multiple odontogenic keratocysts: an other observation. Introduction. The odontogenic keratocyst is an important part in the clinical features of basal cell nevus syndrome or Gorlin and Goltz syndrome. Mutation of the PTCH gene is partly responsible; the partial expression may be at the origin of the appearance of multiple recurrent keratocysts. Observation. It was reported throughout this article the case of a 54-year-old patient with no symptom of Gorlin and Goltz syndrome but reveals, however multiple cysts on the full extent of the mandible. The treatment consisted of enucleation of cystic cavities in several stages, pathological examination of the surgical specimen confirmed the diagnosis of multiple keratocyst. The follow over 24 months shows no signs of recurrence. Discussion. Sometimes, multiple keratocysts can be seen without basal cell nevus syndrome.
\end{abstract}

\section{Introduction}

Le kératokyste odontogénique (KO) ou kyste épidermoïde dérive de la lame dentaire ou de ses vestiges, et de l'assise basale de l'épithélium buccal. Il représente entre 10 et $20 \%$ de l'ensemble des lésions kystiques des maxillaires et il est classé par l'OMS en $3^{\mathrm{e}}$ position par ordre de fréquence dans la catégorie des lésions kystiques. Le KO atteint préférentiellement les hommes entre 40 et 50 ans et il siège dans $70 \%$ des cas dans la région de l'angle et du ramus de la mandibule.

Le $\mathrm{K} 0$ est un des éléments caractéristiques de la naevomatose basocellulaire (NBC) ou syndrome de Gorlin et Goltz. Ce syndrome est associé à une mutation du gène $\mathrm{PCTH}$; une expression partielle de ce gène peut être responsable uniquement de l'apparition de KO multiples récidivants [1, 2].
Nous rapportons le cas d'un patient présentant des $\mathrm{K} 0$ multiples non syndromiques qui, après énucléation chirurgicale, n'ont pas montré de signes de récidive au terme de 2 années d'un suivi, clinique et radiologique, régulier.

\section{Observation}

Il s'agit d'un patient âgé de 54 ans en bon état général, sans antécédents médico-chirurgicaux, qui a consulté pour un suintement sur le site d'extraction de la 36 ; l'extraction a été faite 2 ans auparavant. À l'examen exobuccal, on note l'absence d'asymétrie faciale et d'adénopathies. L'examen endobuccal met en évidence une soufflure des corticales linguale et vestibulaire à hauteur de 34 et 35 , et une fistule non productive sur le site d'extraction de la 36 (Fig. 1). Il n'y avait aucun point

\footnotetext{
*Correspondance : haitamisofia@yahoo.com
} 


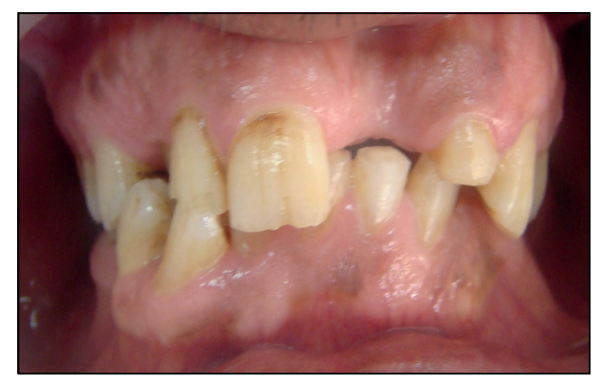

Fig. 1. Vue endobuccale montrant la tuméfaction vestibulaire à hauteur de 34 et 35 .

Fig. 1. Intraoral view showing a vestibular tumefaction at the level of 34 and 35.

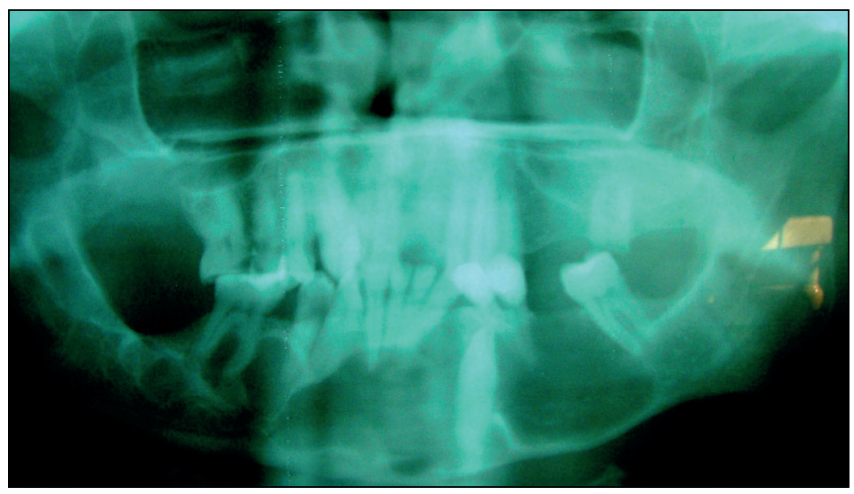

$\mathbf{a}$

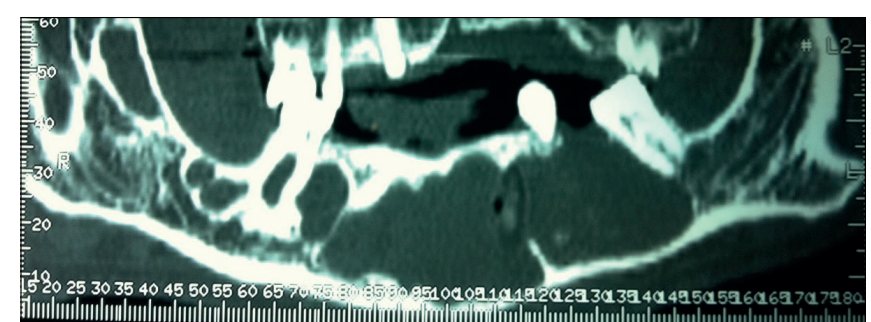

b

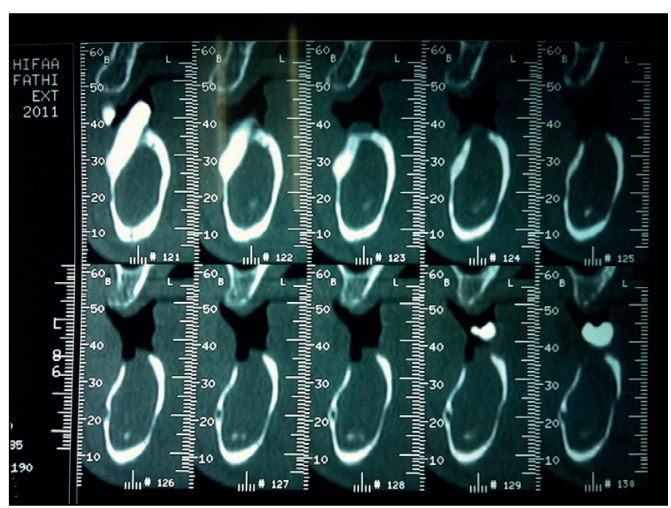

c

Fig. 2. a, b, c. Radiographie panoramique et dentascan montrant des lacunes radioclaires intéressant tout le corps de la mandibule. Fig. $2 a, b$, c. Panoramic radiography and CT-scan showing radiolucent gaps involving the mandible.

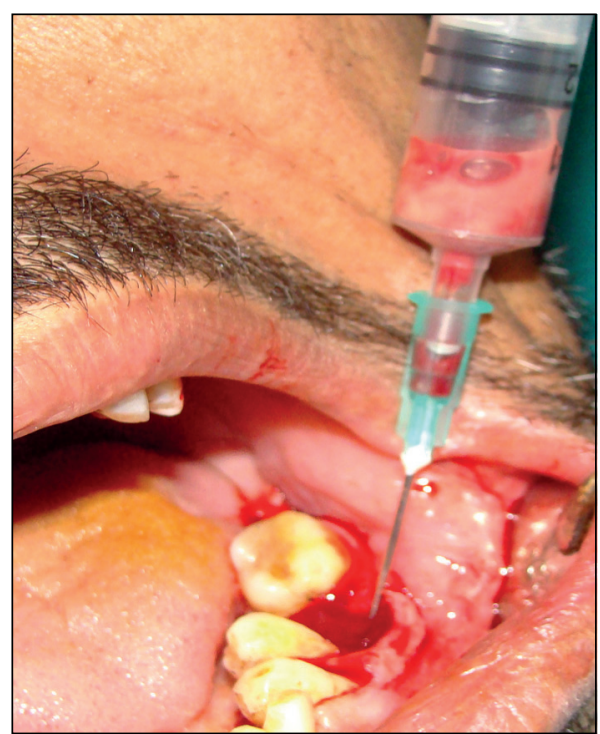

Fig. 3. Ponction dans la région du site d'extraction de la 36 ramenant un liquide épais et purulent.

Fig. 3. Puncture of a thick purulent fluid in the extraction site of 36.

douloureux à la palpation. Le test de vitalité est positif pour toutes les dents mandibulaires.

L'examen radiologique panoramique, complété par un examen tomodensitométrique, montrait des lésions lacunaires radioclaires, multigéodiques bien limitées, s'étendant de la région molaire inferieure gauche jusqu'à la branche montante droite. On notait également la présence de la 33 incluse. (Fig. 2a-2c).

En se basant sur les données cliniques et radiographiques, on a évoqué plusieurs diagnostics : un kyste folliculaire en rapport avec la 33 incluse, un améloblastome en raison de l'aspect multigéodique de la lésion, des $\mathrm{K} 0$ multiples non syndromiques tout en écartant l'hypothèse d'un syndrome de Gorlin et Goltz. En effet, l'absence de carcinome baso-cellulaire, d'anomalies osseuses, oro-faciales ou dermatologiques, d'un défaut de croissance ou d'une hyperlaxité ligamentaire orientent vers une affection non syndromique.

La ponction dans la région du site d'extraction de la 36 a ramené un liquide épais, purulent et jaunâtre (Fig. 3). Le traitement a consisté en l'énucléation chirurgicale de 3 lésions kystiques en 2 temps opératoires, à un mois d'intervalle.

L'examen histologique des pièces opératoires a montré que les parois kystiques comportaient un revêtement malpighien hyperplasique et papillomateux avec une assisse de cellules cylindriques, palissadiques, et une parakératose de surface ; tous ces éléments sont en faveur du diagnostic de K0 (Fig. 4).

Les contrôles cliniques et radiologiques effectués pendant les 2 ans qui ont suivi l'intervention ont montré une reminéralisation osseuse des lacunes résiduelles. On notait aussi l'absence de séquelles neurologiques. 


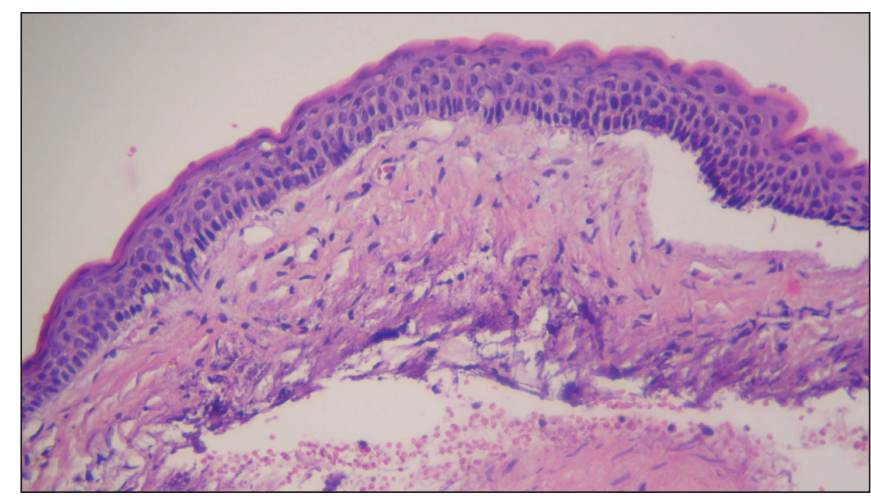

Fig. 4. Aspect microscopique de la paroi kystique. Fig. 4. Microscopic aspect of the cyst wall.

\section{Discussion}

En 2005, le groupe de travail de l'OMS a classé le KO parakératinisé dans la catégorie des tumeurs bénignes en le différenciant de sa variante orthokératinisée toujours considérée comme un $\mathrm{KO}$.

Le $\mathrm{KO}$ est une tumeur à prédominance masculine $(60 \%)$, localisée plus fréquemment à la mandibule (70\%), données retrouvées dans notre cas. La forme multiple est souvent associée à des syndromes tels que le syndrome oro-digito-facial, le syndrome de Noonan, le syndrome d'Ehlers-Danlos ou le syndrome de Simpson-Golabi-Behmel mais le plus fréquent reste la NBC ou syndrome de Gorlin et Goltz [1].

Dans le cas de K0 multiples et volumineux, comme dans notre cas, la probabilité du syndrome de Gorlin et Goltz doit être évoquée en présence d'anomalies associées comme une bifidité costale, un hypertélorisme, un brachymétacarpisme, un retard mental et des naevus basocellulaires multiples [2].

Les $\mathrm{KO}$ et les carcinomes baso-cellulaires survenant dans un contexte de NBC sont associés à une mutation du gène $\mathrm{PCTH}$ dans les cellules précurseurs de ces lésions ainsi qu'à une perte de l'hétérozygotisme. Depuis la mise en évidence de la relation de causalité entre le gène PCTH et la NBC, plus de 60 nouvelles mutations ont été découvertes [3-5] et plus de $40 \%$ des cas présentent une nouvelle mutation. En 1999, Gorlin a affirmé avoir retrouvé la même altération de ce gène dans des cas de neuroblastome, de carcinome baso-cellulaire et des K0 n'appartenant pas à une forme syndromique [in 6]. La grande variabilité de l'expression du gène PCTH soulève la question de sa spécificité pour le diagnostic différentiel des KO multiples non syndromiques [6].

Seul l'examen histologique permet de poser le diagnostic de certitude devant un épithélium malpighien pluristratifié, parakératinisé, associé à des reliquats épithéliaux intra-muraux et des kystes satellites, Ces caractéristiques histologiques permettraient de différencier les kératokystes associés au syndrome de Gorlin et Goltz des kératokystes isolés [3, 7].
L'immunohistochimie permet d'écarter, en cas de doute, les autres lésions kystiques, principalement le kyste dentigère ou le kyste radiculaire ; cet examen met en évidence la présence d'indicateurs spécifiques du K0, à savoir l'expression des cytokératines 17 et 19 et une expression élevée du PCNA et du Ki67 qui témoignent du caractère agressif et récidivant du K0 [8].

Le traitement est chirurgical. Il consiste à réaliser une énucléation complète, éventuellement associée à l'application de la solution de Carnoy dans le but de diminuer le taux de récidive qui varie entre 9 et $25 \%$ à 5 ans. Certains auteurs attribuent le caractère récidivant des $\mathrm{K} 0$ associés dans la NBC par rapport aux KO isolés à un turnover plus important et/ou la présence de kystes filles non éliminés lors de l'intervention chirurgicale. Un suivi à long terme est fortement conseillé afin de dépister précocement toute récidive [9].

Pour Lo Muziol et al., les KO multiples constituent une manifestation précoce de la NBC et ils sont observés chez des patients jeunes [10]. Dans le cas rapporté, le patient est âgé de 54 ans et il ne présente aucun signe évocateur de NBC. On ne note également aucune récidive après 24 mois. L'ensemble de ces éléments amènent à confirmer le diagnostic de $\mathrm{KO}$ multiples non syndromiques, et d'ajouter ce cas aux 3 autres rapportés dans la littérature $[3,6,11]$.

\section{Conflits d'intérêt : aucun}

\section{Références}

1. Brannon RB. The odontogenic keratocyst. A clinicopathologic study of 312 cases. Part 1. Clinical features. Oral Surg Oral Med Oral Pathol 1976;42:54-72.

2. Ljubenović M, Ljubenović D, Binić I, Jovaoivić D, Stanojević M. Gorlin-Goltz syndrome. Acta Dermatovenereol Alp Panonica Adriat 2007;16:166-9.

3. Bartake AR, Shreekanth NG, Prabh S, Gopalkrishnan K. Nonsyndromic recurrent multiple odontogenic keratocysts: a case report . J Dent (Tehran) 2011;8:96-100.

4. Marsh A, Wicking C, Wainwright B, Chenevix-Trench G. DHPLC analysis of patients with nevoid basal cell carcinoma syndrome reveals novel PCTH missense mutations in the sterol-sensing domain. Hum Mutat 2005;26:283.

5. Ortega Garcia de Amezaga A, Gracia Arregui 0, Zepeda Nuño S, Acha Sagredo A, Aguirre Urizar JM. Gorlin-Goltz syndrome: clinicopathologic aspects. Med Oral Patol Oral Cir Bucal 2008; 13:e338-43.

6. Auluck A, Suhas S, Pai KM. Multiple odontogenic keratocysts: report of a case. J Can Dent Assoc 2006;72:651-6.

7. Domingez FV, Keszler A. Comparative study of kertaocysts, associated and non-associated with nevoid basal cell carcinoma syndrome. J Oral Pathol 1988;17:39-42.

8. El Murtadi A, Grehan D, Toner M, McCartan BE. Proliferating cell nuclear antigen staining in syndrome and non syndrome 
odontogenic keratocysts. Oral Surg Oral Med Oral Pathol Oral Radiol Endod 1996;81:217-20.

9. Mateus GC, Lanza GH, de Moura PH, Marigo Hde A, Horta MC. Cell prolifération and apoptosis in keratocystic odontogenic tumors. Med Oral Patol Oral Cir Bucal 2008;13:e697-702.
10. Lo Muzio L, Nocini P, Bucci P, Pannone G, Consolo U, Procaccini M. Early diagnosis of nevoid basal cell carcinoma syndrome. J Am Dent Assoc 1999;130:669-74.

11. Parikh NR. Nonsyndromic multiple odontogenic keratocysts: report of case. J Adv Dent Res 2010;1:71-4. 